\title{
Higher-order Fefferman-Poincaré type inequalities and applications
}

Kelei Zhang and Pengcheng Niu*

\section{"Correspondence:} pengchengniu@nwpu.edu.cn Department of Applied Mathematics, Northwestern Polytechnical University, Xi'an, Shaanxi 710129, P.R. China

\begin{abstract}
We establish higher-order Fefferman-Poincaré type inequalities with a potential belonging to an appropriate higher-order Stummel-Kato type class introduced in this paper. As an application, we obtain a priori $L^{p}$ estimates for solutions of higher-order elliptic equations with discontinuous coefficients of small BMO type and a potential belonging to the higher-order Stummel-Kato type class.
\end{abstract}

MSC: Primary 46E35; 35J30; secondary 35B45; 35J10

Keywords: higher-order Fefferman-Poincaré type inequality; higher-order elliptic equation; potential; higher-order Stummel-Kato type class; $L^{p}$ estimate

\section{Introduction and main results}

Fefferman proved in [1] that if a potential $V$ belonging to the classical Morrey space $L^{r, n-2 r}\left(\mathbb{R}^{n}\right)$ with $1<r \leq n / 2$, then there exists a positive constant $c$, independent of $u$, such that

$$
\int_{\mathbb{R}^{n}}|V(x)||u(x)|^{2} d x \leq c \int_{\mathbb{R}^{n}}|\nabla u(x)|^{2} d x, \quad u \in C_{0}^{\infty}\left(\mathbb{R}^{n}\right) .
$$

The result has been extended to many more general settings and applied to study Harnack's inequality, unique continuation for nonnegative solutions and regularity of solutions of elliptic equations (cf. [2-8] etc.). Especially, Schechter [7] deduced a similar inequality for $V \in S\left(\mathbb{R}^{n}\right)$, here the space $S\left(\mathbb{R}^{n}\right)$ is the classical Stummel-Kato class. Let us recall that one says $V \in S\left(\mathbb{R}^{n}\right)(n \geq 3)$ if $V \in L_{\text {loc }}^{1}\left(\mathbb{R}^{n}\right)$, and for any $r>0$,

$$
\varphi_{V}(r):=\sup _{x \in \mathbb{R}^{n}} \int_{B(x, r)}|V(y)||x-y|^{2-n} d y
$$

is finite and

$$
\lim _{r \rightarrow 0^{+}} \varphi_{V}(r)=0,
$$

where $B(x, r)$ is a ball of radius $r$ and center $x$ in $\mathbb{R}^{n}$. Recently, Zamboni [8] introduced a new function space including $S\left(\mathbb{R}^{n}\right)$ in terms of nonlinear Riesz potentials, and also provided a Fefferman-Poincaré inequality extending the result in [7].

(c) 2015 Zhang and Niu. This article is distributed under the terms of the Creative Commons Attribution 4.0 International License (http://creativecommons.org/licenses/by/4.0/), which permits unrestricted use, distribution, and reproduction in any medium, provided you give appropriate credit to the original author(s) and the source, provide a link to the Creative Commons license, and indicate if changes were made. 
Inspired by Zamboni [8], Definition 2.3, we introduce the following higher-order Stummel-Kato type class $S_{p}^{m}\left(\mathbb{R}^{n}\right)$.

Definition 1.1 Let $V \in L_{\text {loc }}^{1}\left(\mathbb{R}^{n}\right)(n \geq 3)$, and let $p>1$ and $m$ be a positive integer with $1 \leq m<n$. For $r>0$, denote

$$
\eta_{V}(r):=\sup _{x \in \mathbb{R}^{n}}\left(\int_{B_{r}(x)} \frac{1}{|x-y|^{n-m}}\left(\int_{B_{r}(x)} \frac{|V(z)|}{|y-z|^{n-m}} d z\right)^{\frac{1}{p-1}} d y\right)^{p-1} .
$$

We say that $V$ belongs to the higher-order Stummel-Kato type class $S_{p}^{m}\left(\mathbb{R}^{n}\right)$, if

$$
\eta_{V}(r)<+\infty, \quad r>0 \text { and } \lim _{r \rightarrow 0^{+}} \eta_{V}(r)=0 .
$$

Here $|\cdot|$ denotes the Euclidean norm and $B_{r}(x)=B(x, r)=\left\{y \in \mathbb{R}^{n}:|x-y|<r\right\}$. Sometimes we call that $\eta_{V}(r)$ is the higher-order Stummel-Kato type modulus of $V$.

Remark 1.2 If $m=1$, the definition above is identical with Definition 2.3 in [8]; if $m=2$, the definition above is the same as Definition 1 in [6] corresponding to the Euclidean case.

We also need the following definitions.

Definition 1.3 ([9]) Let $\Omega$ be an open set in $\mathbb{R}^{n}$. For $p \geq 1$ and $k$ a nonnegative integer, the Sobolev space $W^{k, p}(\Omega)$ consists of all distributions $u$ on $\Omega$ such that $D^{\alpha} u \in L^{p}(\Omega)$ for all multi-index $\alpha$ with $|\alpha| \leq k$. Furthermore, $W^{k, p}(\Omega)$ is a Banach space with the norm

$$
\|u\|_{W^{k, p}(\Omega)}=\left(\int_{\Omega} \sum_{|\alpha| \leq k}\left|D^{\alpha} u\right|^{p} d x\right)^{1 / p}
$$

Here, $\alpha=\left(\alpha_{1}, \ldots, \alpha_{n}\right)$ is a multi-index of order $|\alpha|:=\alpha_{1}+\cdots+\alpha_{n}$. The Banach space $W_{0}^{k, p}(\Omega)$ is the closure of $C_{0}^{\infty}(\Omega)$ in $W^{k, p}(\Omega)$.

We denote $D^{k} u:=\left\{D^{\alpha} u:|\alpha|=k\right\}$, and its norm is defined by

$$
\left|D^{k} u\right|=\left(\sum_{|\alpha|=k}\left|D^{\alpha} u\right|^{2}\right)^{1 / 2}
$$

For convenience, we denote by $M B(x, r)(M>0)$ the ball concentric with $B(x, r)$ of radius $M$ times that of $B(x, r)$.

Definition 1.4 ([10]) A domain $\Omega$ in $\mathbb{R}^{n}$ is said to be a weak Boman chain domain, or a member of $\mathcal{F}(1, M)$, if there exist a constant $M \geq 1$ and a family $\mathcal{F}$ of balls $B \subset \Omega$ such that

(i) $\Omega=\bigcup_{B \in \mathcal{F}^{B}}$;

(ii) $\sum_{B \in \mathcal{F}} \chi_{B}(x) \leq M \chi_{\Omega}(x), x \in \mathbb{R}^{n}$;

(iii) there is a 'central ball' $B_{0} \in \mathcal{F}$ such that for every ball $B \in \mathcal{F}$, there exist a positive integer $k=k(B)$ and a chain $\left\{B_{j}\right\}_{j=0}^{k}$ of balls for which and each $B_{j} \cap B_{j+1}$ contains a ball $D_{j}$ (this ball does not need to be a member of $\mathcal{F}$ ) with $B_{j} \cup B_{j+1} \subset M D_{j}$;

(iv) $B \subset M B_{j}$ for all $j=0,1, \ldots, k(B)$. 
Let us note that if the hypothesis (ii) is replaced by

$$
\sum_{B \in \mathcal{F}} \chi_{\tau B}(x) \leq M \chi_{\Omega}(x), \quad x \in \mathbb{R}^{n}, \tau>1 \text { is a constant }
$$

then $\Omega$ is said to be a Boman chain domain, $c f$. [11, 12]. The classes $\mathcal{F}(1, M)$ contain many important types of domains in $\mathbb{R}^{n}$, for instance, balls, cubes, and John domains ( $c f$. $[11,13,14])$.

Based on the class $S_{p}^{m}$, we establish the following higher-order Fefferman-Poincaré type inequality with the aid of the method in $[4,8]$ proving the Fefferman-Poincaré type inequality for the case $m=1$. It is interesting that the $m$ th order derivatives arise in this setting.

Theorem 1.5 Let $\Omega$ be a weak Boman domain, $p>1$, and $m$ be a positive integer with $1 \leq m<n$. Assume $V \in S_{p}^{m}\left(\mathbb{R}^{n}\right)$, then for any $u \in W^{m, p}(\Omega)$, there exists a polynomial $P_{B_{0}}(x)$ of order less than $m$ such that

$$
\int_{\Omega}\left|u(x)-P_{B_{0}}(x)\right|^{p}|V(x)| d x \leq c \eta_{V}\left(2 M r_{B_{0}}\right) \int_{\Omega}\left|D^{m} u(x)\right|^{p} d x,
$$

where the positive constant $c$ is independent of $u, B_{0}$ is the central ball of radius $r_{B_{0}}$ in $\Omega$, and the constant $M$ is in Definition 1.4.

When $u$ is a distribution with compact support in $\Omega$, we have the following.

Theorem 1.6 Under the assumptions of Theorem 1.5, for any $u \in W_{0}^{m, p}(\Omega)$, there exists a positive constant $c$ independent of $u$ such that

$$
\int_{\Omega}|u(x)|^{p}|V(x)| d x \leq c \eta_{V}\left(2 M r_{B_{0}}\right) \int_{\Omega}\left|D^{m} u(x)\right|^{p} d x .
$$

\section{Remark 1.7}

(i) In the special case when $m=1$, similar results were obtained in $[4,8]$; when $m=2$ and $\Omega=B(x, r)$, (1.1) and (1.2) have been obtained in [6].

(ii) The higher-order Fefferman-Poincaré type inequalities on stratified groups can also be proved, because of the higher-order representation formulas proved by Lu and Wheeden $[10,15]$.

In the rest of this paper, we will show some applications of the above results to regularity of solutions to the higher-order elliptic equations with a potential. Let us consider the equation

$$
\sum_{|\alpha| \leq 2 k} a_{\alpha}(x) D^{\alpha} u(x)+V(x) u(x)-\lambda u(x)=f(x), \quad x \in \mathbb{R}^{n},
$$

where $k$ is a positive integer with $1 \leq k<n / 2, V \in S_{p}^{2 k}\left(\mathbb{R}^{n}\right)$ for $p>1$, and the coefficients $a_{\alpha}(x)$ satisfy

$$
(-1)^{k-1} \sum_{|\alpha|=2 k} a_{\alpha}(x) \xi^{\alpha} \geq \Lambda_{1}, \quad \xi \in \mathbb{S}^{n-1} \subset \mathbb{R}^{n}
$$


and

$$
\sum_{|\alpha| \leq 2 k}\left|a_{\alpha}(x)\right| \leq \Lambda_{2}
$$

for all $x \in \mathbb{R}^{n}$ and positive constants $\Lambda_{1}, \Lambda_{2}$. In addition, we assume that the leading coefficients $a_{\alpha}(x)(|\alpha|=2 k)$ are in BMO space and their semi-norms are small enough. More precisely, we recall the following definition.

Definition 1.8 (Small BMO condition, $c f$. [16]) We say that the coefficients $a_{\alpha}(x)$ satisfy $(\delta, R)$-vanishing condition if for given $\delta>0$, there exists $R>0$ such that

$$
\sup _{0<r \leq R x \in \mathbb{R}^{n}} \sup _{n}\left|B_{r}(x)\right|^{-1} \int_{B_{r}(x)}\left|a_{\alpha}(y)-\overline{\left(a_{\alpha}\right)} B_{r}(x)\right| d y \leq \delta,
$$

where

$$
{\overline{\left(a_{\alpha}\right)}}_{B_{r}(x)}=\left|B_{r}(x)\right|^{-1} \int_{B_{r}(x)} a_{\alpha}(y) d y .
$$

When $k=1$ and $V$ in general is not bounded, or $k>1$ and $V \in L^{\infty}\left(\mathbb{R}^{n}\right)$, regularity for the elliptic equation (1.3) has been studied by many authors, $c f$. [16-19] and so forth. Here we are concerned with (1.3) for the case $1<k<n / 2$ and a singular potential $V$ in $S_{p}^{m}\left(\mathbb{R}^{n}\right)$.

Our result from (1.3) is the following.

Theorem 1.9 Let $p \in(1, \infty), k$ be a positive integer with $1<k<n / 2$. There exist a positive $\lambda_{0}=\lambda_{0}\left(n, \Lambda_{1}, \Lambda_{2}, p\right)$ and a small $\delta=\delta\left(n, \Lambda_{1}, \Lambda_{2}, p\right)>0$ such that for the coefficients $a_{\alpha}(x)$ satisfying (1.4)-(1.5) and ( $\delta, R)$-vanishing condition with $|\alpha|=2 k$, for $V^{p} \in S_{p}^{2 k}\left(\mathbb{R}^{n}\right)$ and $f \in$ $L^{p}\left(\mathbb{R}^{n}\right)$, if $u \in W^{2 k, p}\left(\mathbb{R}^{n}\right)$ solves equation (1.3), then

$$
\sum_{|\alpha| \leq 2 k}\left\|D^{\alpha} u\right\|_{L^{p}\left(\mathbb{R}^{n}\right)}+\|V u\|_{L^{p}\left(\mathbb{R}^{n}\right)} \leq c\|f\|_{L^{p}\left(\mathbb{R}^{n}\right)}
$$

provided $\lambda \geq \lambda_{0}$, where the positive constant $c$ is independent of $u$ and $f$.

This paper is organized as follows. In Section 2 we first give an example showing the class $S_{p}^{m}\left(\mathbb{R}^{n}\right)$ contains some nontrivial singular functions, and then prove Theorems 1.5 and 1.6 using the higher-order representation formulas by $\mathrm{Lu}$ and Wheeden in $[10,15]$. The proof of Theorem 1.9 is given in Section 3 based on results in previous section and $L^{p}$ estimates for the higher-order elliptic equations without potentials in $[16,18]$.

Dependence of constants Throughout this paper, the letter $c$ denotes a positive constant which may vary from line to line.

\section{Proofs of Theorems 1.5 and 1.6}

Before giving the proofs of Theorems 1.5 and 1.6, we first show that the higher-order Stummel-Kato type class $S_{p}^{m}\left(\mathbb{R}^{n}\right)(p>1,1 \leq m<n)$ is nonempty. Clearly, $L^{\infty}\left(\mathbb{R}^{n}\right) \subset S_{p}^{m}\left(\mathbb{R}^{n}\right)$ and, in general, the class $S_{p}^{m}\left(\mathbb{R}^{n}\right)$ involves some singular potentials. For example, the function

$$
V(x)=\frac{1}{\left.|x|^{m}|\log | x\right|^{2 m}} \chi_{B\left(0, e^{-2}\right)}(x) \quad \text { for } p>1 \text { and } 1 \leq m<n,
$$


where $\chi_{B\left(0, e^{-2}\right)}$ is the characteristic function of $B\left(0, e^{-2}\right)$, belongs to $S_{p}^{m}\left(\mathbb{R}^{n}\right)$. To illustrate it, we need to show that

$$
\eta_{V}(r)=\sup _{x \in \mathbb{R}^{n}}\left(\int_{B_{r}(x)} \frac{1}{|x-y|^{n-m}}\left(\int_{B_{r}(x)} \frac{|z|^{-m}|\log | z||^{-2 m}}{|y-z|^{n-m}} \chi_{B_{e^{-2}}(0)}(z) d z\right)^{\frac{1}{p-1}} d y\right)^{p-1}
$$

satisfies

(i) $\eta_{V}(r)<\infty, \quad r>0$;

(ii) $\lim _{r \rightarrow 0^{+}} \eta_{V}(r)=0$.

In fact, for $x \in \mathbb{R}^{n}$ and $r>0$, one has

$$
\begin{aligned}
\Phi(x, r) & :=\int_{B(x, r)} \frac{1}{|x-y|^{n-m}}\left(\int_{B(x, r)} \frac{\left.|z|^{-m}|\log | z\right|^{-2 m}}{|y-z|^{n-m}} \chi_{B\left(0, e^{-2}\right)}(z) d z\right)^{\frac{1}{p-1}} d y \\
& \equiv \int_{B(x, r)}|x-y|^{m-n} J^{\frac{1}{p-1}} d y
\end{aligned}
$$

and one has

$$
\begin{aligned}
J \leq & \int_{B(y, 2 r)} \frac{\left.|z|^{-m}|\log | z\right|^{-2 m}}{|y-z|^{n-m}} \chi_{B\left(0, e^{-2}\right)}(z) d z \\
\leq & \int_{\{z:|z|<|y-z|<2 r\}} \frac{|z|^{-m}|\log | z||^{-2 m}}{|y-z|^{n-m}} \chi_{B\left(0, e^{-2}\right)}(z) d z \\
& +\int_{\{z:|z| \geq|y-z|\} \cap B(y, 2 r)} \frac{|z|^{-m}|\log | z||^{-2 m}}{|y-z|^{n-m}} \chi_{B\left(0, e^{-2}\right)}(z) d z \\
\equiv & J_{1}+J_{2} .
\end{aligned}
$$

Using the polar coordinate transformation leads to

$$
\begin{aligned}
J_{1} & \leq \int_{\{z:|z|<2 r\}}|z|^{-n}|\log | z||^{-2 m} \chi_{B\left(0, e^{-2}\right)}(z) d z \\
& \leq \int_{B(0,2 r) \cap B\left(0, e^{-2}\right)}|z|^{-n}|\log | z||^{-2 m} d z \\
& =c(n) \int_{0}^{\sigma} \frac{s^{n-1}}{s^{n}(-\log s)^{2 m}} d s \\
& =c(n, m)(-\log \sigma)^{1-2 m},
\end{aligned}
$$

where $\sigma=\min \left\{2 r, e^{-2}\right\}$. Since the function $t^{-m}(-\log t)^{-2 m}$ is decreasing in $\left(0, e^{-2}\right)$, one infers

$$
\begin{aligned}
J_{2} & =\int_{\left\{z:|y-z| \leq|z|<e^{-2}\right\} \cap B(y, 2 r)}|z|^{-m}|\log | z||^{-2 m}|y-z|^{m-n} d z \\
& \leq \int_{B\left(y, e^{-2}\right) \cap B(y, 2 r)}|y-z|^{-n}|\log | y-z||^{-2 m} d z
\end{aligned}
$$




$$
\begin{aligned}
& =\int_{B(0, \sigma)}|z|^{-n}|\log | z||^{-2 m} d z \\
& =c(n, m)(-\log \sigma)^{1-2 m} .
\end{aligned}
$$

Combining $J_{1}$ and $J_{2}$, we have

$$
\begin{aligned}
\Phi(x, r) & \leq c(n, m) \frac{1}{(-\log \sigma)^{(2 m-1) /(p-1)}} \int_{B(x, r)} \frac{1}{|x-y|^{n-m}} d y \\
& \leq c(n, m) \frac{1}{(-\log \sigma)^{(2 m-1) /(p-1)}} \int_{0}^{r} \frac{s^{n-1}}{s^{n-m}} d s \\
& \leq c(n, m) \frac{1}{(-\log \sigma)^{(2 m-1) /(p-1)}} r^{m} .
\end{aligned}
$$

Therefore,

$$
\begin{aligned}
\eta_{V}(r) & =\sup _{x \in \mathbb{R}^{n}} \Phi(x, r)^{p-1} \\
& \leq c(n, m, p)(-\log \sigma)^{1-2 m} r^{m(p-1)} \\
& = \begin{cases}c(n, m, p) 2^{1-2 m} r^{m(p-1)}, & r \geq e^{-2} / 2, \\
c(n, m, p)(-\log (2 r))^{1-2 m} r^{m(p-1)}, & r<e^{-2} / 2,\end{cases}
\end{aligned}
$$

and it proves (i) and (ii).

Now we devote ourselves to proving Theorems 1.5 and 1.6. Lu and Wheeden [10,15] derived various higher-order integral representation formulas on Carnot groups and applied them to prove some Sobolev type embedding theorems. Since the Euclidean space $\mathbb{R}^{n}$ is a special case of Carnot groups, the results in $[10,15]$ are also true in $\mathbb{R}^{n}$. Here, we state these formulas in $[10,15]$ corresponding to $\mathbb{R}^{n}$ and apply to prove (1.1) and (1.2).

Lemma 2.1 $([10,15])$ Let $\Omega$ be a weak Boman chain domain in $\mathbb{R}^{n}$ with a central ball $B_{0}$, and $m$ be a positive integer with $1 \leq m<n$. If $u \in W^{m, 1}(\Omega)$, then there exists a polynomial $P_{B_{0}}(x)$ of order less than $m$ such that for a.e. $x \in \Omega$,

$$
\left|u(x)-P_{B_{0}}(x)\right| \leq c \int_{\Omega}|x-y|^{m-n}\left|D^{m} u(y)\right| d y,
$$

where the positive constant $c$ is independent of $u, x$, and $\Omega$. Moreover, if $u \in W_{0}^{m, 1}(\Omega)$, then

$$
|u(x)| \leq c \int_{\Omega}|x-y|^{m-n}\left|D^{m} u(y)\right| d y .
$$

Proof of Theorem 1.5 Applying (2.1), Fubini's theorem, and Hölder's inequality, we obtain

$$
\begin{aligned}
& \int_{\Omega}\left|u(x)-P_{B_{0}}(x)\right|^{p}|V(x)| d x \\
& \quad \leq c \int_{\Omega}|V(x)|\left|u(x)-P_{B_{0}}(x)\right|^{p-1}\left(\int_{\Omega}\left|D^{m} u(y)\right||x-y|^{m-n} d y\right) d x \\
& \quad=c \int_{\Omega}\left|D^{m} u(y)\right|\left(\int_{\Omega}|V(x)|\left|u(x)-P_{B_{0}}(x)\right|^{p-1}|x-y|^{m-n} d x\right) d y
\end{aligned}
$$




$$
\begin{aligned}
& \leq c\left(\int_{\Omega}\left|D^{m} u(y)\right|^{p} d y\right)^{\frac{1}{p}} \\
& \quad \times\left(\int_{\Omega}\left(\int_{\Omega} \frac{|V(x)|\left|u(x)-P_{B_{0}}(x)\right|^{p-1}}{|x-y|^{n-m}} d x\right)^{\frac{p}{p-1}} d y\right)^{\frac{p-1}{p}} \\
& \equiv c\left(\int_{\Omega}\left|D^{m} u(y)\right|^{p} d y\right)^{\frac{1}{p}} \cdot I .
\end{aligned}
$$

Observing

$$
\begin{aligned}
& \int_{\Omega}|V(x)|\left|u(x)-P_{B_{0}}(x)\right|^{p-1}|x-y|^{m-n} d x \\
& \quad \leq\left(\int_{\Omega} \frac{|V(z)|}{|z-y|^{n-m}} d z\right)^{\frac{1}{p}}\left(\int_{\Omega}\left|u(x)-P_{B_{0}}(x)\right|^{p} \frac{|V(x)|}{|x-y|^{n-m}} d x\right)^{\frac{p-1}{p}},
\end{aligned}
$$

and noting $\Omega \subset M B_{0}$ from (iv) of Definition 1.4 , we deduce

$$
\begin{aligned}
I^{p /(p-1)} \leq & \int_{\Omega}\left(\int_{\Omega} \frac{|V(z)|}{|z-y|^{n-m}} d z\right)^{\frac{1}{p-1}}\left(\int_{\Omega}\left|u(x)-P_{B_{0}}(x)\right|^{p} \frac{|V(x)|}{|x-y|^{n-m}} d x\right) d y \\
= & \int_{\Omega}\left|u(x)-P_{B_{0}}(x)\right|^{p}|V(x)| \\
& \times\left(\int_{\Omega} \frac{1}{|x-y|^{n-m}}\left(\int_{\Omega} \frac{|V(z)|}{|z-y|^{n-m}} d z\right)^{\frac{1}{p-1}} d y\right) d x \\
\leq & \int_{\Omega}\left|u(x)-P_{B_{0}}(x)\right|^{p}|V(x)| \\
& \times\left(\int_{M B_{0}} \frac{1}{|x-y|^{n-m}}\left(\int_{M B_{0}} \frac{|V(z)|}{|z-y|^{n-m}} d z\right)^{\frac{1}{p-1}} d y\right) d x \\
\leq & \int_{\Omega}\left|u(x)-P_{B_{0}}(x)\right|^{p}|V(x)| \\
& \times\left(\int_{B\left(x, 2 M r_{B_{0}}\right)} \frac{1}{|x-y|^{n-m}}\left(\int_{B\left(x, 2 M r_{B_{0}}\right)} \frac{|V(z)|}{|z-y|^{n-m}} d z\right)^{\frac{1}{p-1}} d y\right) d x \\
\leq & \left(\eta_{V}\left(2 M r_{B_{0}}\right)\right)^{\frac{1}{p-1}} \int_{\Omega}\left|u(x)-P_{B_{0}}(x)\right|^{p}|V(x)| d x .
\end{aligned}
$$

Thus,

$$
\begin{aligned}
& \int_{\Omega}\left|u(x)-P_{B_{0}}(x)\right|^{p}|V(x)| d x \\
& \quad \leq c\left(\eta_{V}\left(2 M r_{B_{0}}\right)\right)^{\frac{1}{p}}\left(\int_{\Omega}\left|u(x)-P_{B_{0}}(x)\right|^{p}|V(x)| d x\right)^{\frac{p-1}{p}}\left(\int_{\Omega}\left|D^{m} u(y)\right|^{p} d y\right)^{\frac{1}{p}} .
\end{aligned}
$$

It implies (1.1).

Proof of Theorem 1.6 By using (2.2) and repeating the argument for (1.1), it immediately get (1.2). 


\section{Proof of Theorem 1.9}

The following $L^{p}$ estimates for the higher-order elliptic equations without potentials are well known, $c f$. $[16,18]$.

Lemma 3.1 Consider the equation

$$
\sum_{|\alpha| \leq 2 k} a_{\alpha}(x) D^{\alpha} u-\lambda u=f \quad \text { in } \mathbb{R}^{n}
$$

Let $p>1$. There exist a positive $\lambda_{0}=\lambda_{0}\left(n, \Lambda_{1}, \Lambda_{2}, p\right)$ and a small $\delta=\delta\left(n, \Lambda_{1}, \Lambda_{2}, p\right)>0$ so that for the coefficients $a_{\alpha}(x)$ satisfying (1.4)-(1.5) and $(\delta, R)$-vanishing condition for $|\alpha|=$ $2 k$, and for $f \in L^{p}\left(\mathbb{R}^{n}\right)$, if $u \in W^{2 k, p}\left(\mathbb{R}^{n}\right)$ solves equation (3.1), then

$$
\sum_{|\alpha| \leq 2 k}\left\|D^{\alpha} u\right\|_{L^{p}\left(\mathbb{R}^{n}\right)} \leq c\|f\|_{L^{p}\left(\mathbb{R}^{n}\right)}
$$

provided $\lambda \geq \lambda_{0}$, where the positive constant $c$ is independent of $u$ and $f$.

Proof of Theorem 1.9 Let $r_{0}$ be a positive constant which will be chosen later. By the theorem of the partition of unity (e.g., $f f$. [17] or [20], p.66), there is a sequence of nonnegative functions $\left\{\varphi_{i}\right\}_{i=1}^{\infty}$ in $\mathbb{R}^{n}$ such that

$$
0 \leq \varphi_{i}(x) \leq 1 ; \quad \varphi_{i} \in C_{0}^{\infty}\left(B\left(z_{i}, r_{0}\right)\right) ; \quad \sum_{i=1}^{\infty} \varphi_{i}(x)=1, \quad x \in \mathbb{R}^{n}
$$

and the family of balls $B\left(z_{i}, r_{0}\right)$ has the finite overlapping property. One may obviously note $\varphi_{i} u \in W^{2 k, p}\left(\mathbb{R}^{n}\right)$ and $\operatorname{supp}\left(\varphi_{i} u\right) \subset B\left(z_{i}, r_{0}\right)$ and the fact that the ball is a special weak Boman domain. Hence (1.2) also holds for $\varphi_{i} u \in W_{0}^{2 k, p}\left(B\left(z_{i}, r_{0}\right)\right)$. Since $f \in L^{p}\left(\mathbb{R}^{n}\right)$ and $\sum_{|\alpha| \leq 2 k} a_{\alpha}(x) D^{\alpha} u \in L^{p}\left(\mathbb{R}^{n}\right)$ from the boundedness of $a_{\alpha}(x)$, it follows from (1.3) that $V u \in L^{p}\left(\mathbb{R}^{n}\right)$. Thus,

$$
\begin{aligned}
\int_{\mathbb{R}^{n}}|V u|^{p} d x & =\int_{\mathbb{R}^{n}}\left|\sum_{i}\left(V \varphi_{i} u\right)\right|^{p} d x \\
& \leq c \sum_{i} \int_{B\left(z_{i}, r_{0}\right)}\left|V\left(\varphi_{i} u\right)\right|^{p} d x \\
& \leq c \eta_{V^{p}}\left(2 r_{0}\right) \sum_{i} \int_{B\left(z_{i}, r_{0}\right)}\left|D^{2 k}\left(\varphi_{i} u\right)\right|^{p} d x \\
& \leq c \eta_{V^{p}}\left(2 r_{0}\right) \sum_{i} \int_{B\left(z_{i}, r_{0}\right)}\left(\sum_{|\alpha|=2 k}\left|D^{\alpha}\left(\varphi_{i} u\right)\right|\right)^{p} d x \\
& \leq c \eta_{V^{p}}\left(2 r_{0}\right) \sum_{i} \int_{B\left(z_{i}, r_{0}\right)} \sum_{|\alpha| \leq 2 k}\left|D^{\alpha} u\right|^{p} d x \\
& \leq c \eta_{V^{p}}\left(2 r_{0}\right) \int_{\mathbb{R}^{n}} \sum_{|\alpha| \leq 2 k}\left|D^{\alpha} u\right|^{p} d x .
\end{aligned}
$$


By Lemma 3.1 and (3.3), we have

$$
\begin{aligned}
& \sum_{|\alpha| \leq 2 k}\left\|D^{\alpha} u\right\|_{L^{p}\left(\mathbb{R}^{n}\right)}+\|V u\|_{L^{p}\left(\mathbb{R}^{n}\right)} \\
& \quad \leq c\left(\|f-V u\|_{L^{p}\left(\mathbb{R}^{n}\right)}+\|V u\|_{L^{p}\left(\mathbb{R}^{n}\right)}\right) \\
& \quad \leq c\left(\|f\|_{L^{p}\left(\mathbb{R}^{n}\right)}+\|V u\|_{L^{p}\left(\mathbb{R}^{n}\right)}\right) \\
& \quad \leq c\|f\|_{L^{p}\left(\mathbb{R}^{n}\right)}+c \eta_{V^{p}}\left(2 r_{0}\right)^{\frac{1}{p}} \sum_{|\alpha| \leq 2 k}\left\|D^{\alpha} u\right\|_{L^{p}\left(\mathbb{R}^{n}\right)} .
\end{aligned}
$$

Choosing $r_{0}>0$ such that $c \eta_{V^{p}}\left(2 r_{0}\right)^{1 / p} \leq 1 / 2$, (1.6) is obtained.

\section{Competing interests}

The authors declare that they have no competing interests.

\section{Authors' contributions}

Both authors contributed equally to the writing of this paper. Both authors read and approved the final manuscript.

\section{Acknowledgements}

The authors are very grateful to the anonymous reviewers for their valuable comments and suggestions to improve the expressions. This work was supported by the National Natural Science Foundation of China (Grant No. 11271299), and the Fundamental Research Funds for the Central Universities (Grant No. 3102015ZY069).

\section{Received: 18 April 2015 Accepted: 29 November 2015 Published online: 09 December 2015}

\section{References}

1. Fefferman, C: The uncertainty principle. Bull. Am. Math. Soc. 9(2), 129-206 (1983)

2. Chiarenza, F, Frasca, M: A remark on a paper by C. Fefferman. Proc. Am. Math. Soc. 108(2), 407-409 (1990)

3. Danielli, D: A Fefferman-Phong inequality and applications to quasilinear subelliptic equations. Potential Anal. 11(4), 387-413 (1999)

4. Di Fazio, G, Zamboni, P: A Fefferman-Poincaré type inequality for Carnot-Carathéodory vector fields. Proc. Am. Math. Soc. 130(9), 2655-2660 (2002)

5. Di Fazio, G, Zamboni, P: Fefferman-Poincaré inequality and regularity for quasilinear subelliptic equations. Lect. Notes Semin. Interdiscip. Mat. 3, 103-122 (2004)

6. Niu, PC, Zhang, KL: High order Fefferman-Phong type inequalities in Carnot groups and regularity for degenerate elliptic operators plus a potential. Abstr. Appl. Anal. 2014, Article ID 274859 (2014)

7. Schechter, M: Spectra of Partial Differential Operators. North-Holland, Amsterdam (1986)

8. Zamboni, P: Unique continuation for nonnegative solutions of quasilinear elliptic equations. Bull. Aust. Math. Soc. 64(1), 149-156 (2001)

9. Gilbarg, D, Trudinger, NS: Elliptic Partial Differential Equations of Second Order. Springer, Berlin (2001)

10. Lu, GZ, Wheeden, R: High order representation formulas and embedding theorems on stratified groups and generalizations. Stud. Math. 142(2), 101-133 (2000)

11. Boman, J: $L^{p}$-Estimates for very strongly elliptic systems. Technical report 29, Department of Mathematics, University of Stockholm, Sweden (1982)

12. Iwaniec, T, Nolder, CA: Hardy-Littlewood inequality for quasi-regular mappings in certain domains in $\mathbb{R}^{n}$. Ann. Acad. Sci. Fenn., Ser. A I Math. 10(1), 267-282 (1985)

13. Buckley, S, Koskela, P, Lu, GZ: Boman equals John. In: Proc. of the 16th Nevanlinna Coll. (Joensuu, 1995), pp. 91-99. de Gruyter, Berlin (1996)

14. Garofalo, N, Nhieu, DM: Isoperimetric and Sobolev inequalities for Carnot-Carathéodory spaces and the existence of minimal surfaces. Commun. Pure Appl. Math. 49(10), 1081-1144 (1996)

15. Lu, GZ, Wheeden, R: Simultaneous representation and approximation formulas and high-order Sobolev embedding theorems on stratified groups. Constr. Approx. 20(4), 647-668 (2004)

16. Wang, LH, Yao, FP: Higher-order non-divergence elliptic and parabolic equations in Sobolev spaces and Orlicz spaces. J. Funct. Anal. 262(8), 3495-3517 (2012)

17. Bramanti, M, Brandolini, L, Harboure, E, Viviani, B: Global W'2,p estimates for nondivergence elliptic operators with potentials satisfying a reverse Hölder condition. Ann. Mat. Pura Appl. 191(2), 339-362 (2012)

18. Dong, $\mathrm{HJ}$, Kim, D: On the $L^{p}$-solvability of higher order parabolic and elliptic systems with BMO coefficients. Arch Ration. Mech. Anal. 199(3), 889-941 (2011)

19. Shen, ZW: $L^{p}$ Estimates for Schrödinger operators with certain potentials. Ann. Inst. Fourier (Grenoble) 45(2), 513-546 (1995)

20. Chen, WH: An Introduction to Differentiable Manifold. Higher Education Press of China, Beijing (2001) 